\title{
LXII. \\ Ueber die Darstellung von Aetherarten, durch Einwirkung von Kali auf einige Balsame.
}

\author{
Von \\ E. A. Scharling.
}

(Nitgetheilt voin Verfasser aus der Oversigt over det Kongelige danske Viderskab. Selsk. Forh. 1849 9.)

Die Formel, welche Fremy lïr die Zusammensetzung des Cinnameins aufgestellt hat, $\mathrm{C}_{54} \mathrm{H}_{28} \mathrm{O}_{8}$, kann betrachtet werden als $2\left(\mathrm{C}_{16} \mathrm{H}_{7} \mathrm{O}_{2}\right)+\mathrm{C}_{22} \mathrm{H}_{12} \mathrm{O}_{4} ;$ das erste Glied stell die $\mathrm{Z} 11$ sammensetzung dar, welche $\mathrm{Plantamour}$ für das Cinnamein gegeben, das zweite die des Zimmtäthers, den derselbe aus dem peruviannischen Balsame abgeschieden hat. Da jedoch Plantamour bei seiner Untersuchung Weingeist angewendet hat, so dass man im Zweifel sein konnte, ob nicht dieser die Billung des Aethers veranlasst haben moclite, so unterwarf ich einen Theil peruvianischen Balsam und 2-3 Theile Kalilauge von 1,3 der Destillation, nachdem das Gemenge 24 Stunden gestanden hatte. Das Destillat bestand aus Wasser und zwei ölartigen Körpern, von denen der eine leichter, der andere schwerer als Wasser war. Der letztere war nach dem Trocknen und Ueberdestilliren eine ganz klare, das Licht stark brechende Flüssigkeit, von der Dichte 1,03 (140 C.); ihr Siedepunkt war 205\%. Ihr Geruch war gleich nach der Destillation schwach, wurde jedoch beim Stchen gewürzhaft, wie Zimmtātlıer. In Schnee und Kochsalz abgekühlt bis anf $-15^{\circ} \mathrm{C}$. blicb die ganze Masse llüssig.

Die leichte ölige Flüssigkeit kocht bei etwa $180^{\circ} \mathrm{C}$.; sie war leichter als Wasser, roch :dem Anis äınlich, schmeckte süsslich aromatisch; bis auf $-15^{\circ} \mathrm{C}$. abgekühlt, erstarrte der grösste Theil der Flüssigkeit.

Diese beiden Flüssigkeiten erleiden bei der Destillation eine Veränderung; selbst beim Erwărmen im 0elbade nehmen sie eine schwache weingelbe Farbe an, che sie zu kochen lueginnen. 
Da also diese Flüssigkeiten grosse Achnlichkeiten darboten mit dem Zimmtäther und dem Peruvin, so suchte ich durch Behandlung derselben mit Kalihydrat und Schwefelkohlenstoff ihre Ilentität nachzuweisen.

Ungeachtet $Z$ eis e, der Entdecker der Xanthogensäure, angiebt, dass Aether mit Schwefelkohlenstoff und Kalihydrat kein xanthogensaures Kali liefere, so glaubte ich doch, dass dieses Salz sich bilden könne, wenn man zusammengesetzte Aetherarten mit Kalihydrat und Schwefelkohlenstofr behandele.

Da diese Vermuthung sich vollkommen bestätigte bei andern Aetherarten, namentlich bei dem essigsaurem Aether, so behandelte ich beide aus dem peruvianischen Balsam mil Kali gewonnenen Flüssigkeiten, einzeln mil gepulvertem Kalihydrat und Schwefelkohlenstoff. Ilierbei erstarrten beide Flüssigkeiten; und als nach einiger Zeit eine Probe der Salzmasse zwischen Papier getrocknet, sodann in Wasser gelüst und mit liupfer- und Bleioxydsalzen geprüft wurde, so entstand gelbes xanthogensaures Kupferoxydsalz und weisses xanthogensaures Bleioxydsalz.

Es kann also kein begründeter $Z$ weifel bleiben, dass jenc schwere Flüssigkeit Zimmtäther enthält, die also hier zum ersten Male uhne Anwendung von Weingeist dargestellt worden ist; in wie fern die letztere, leichtere Flïssigkett Peruvin, oder cin Gemenge von Peruvin und Zimmtäther ist, hoffe ich künftig durch die noch nothwendigen Analysen aufzuklären. Dass Peruvin durch Kali nicht zersetzt wird, kann schwerlich als ein Beweis angesehen werden, dass es kein zusammengesetzter Aether sei; durch seine Zersetzung mit Kalihydrat und Schwefelkohlenstoff und durch die dabei gebildeten Stoffe hoffe ich bewcisen zu können, ob das Peruvin eine organische Süure enthallt, deren Formel nach den älteren, übereinstimmenden Formeln desselben wahrscheinlich $\mathrm{C}_{32} \mathrm{H}_{29} \mathrm{O}_{3}$ sein würde, während der Peruvin sellsst aus $\mathrm{C}_{23} \mathrm{H}_{19} \mathrm{O}_{3}+\mathrm{C}_{4} \mathrm{H}_{5} \mathrm{O}$ oder $2\left(\mathrm{C}_{18} \mathrm{H}_{12} \mathrm{O}_{2}\right)$ bestainde.

Wurde ein Theil peruvianischer Balsam mit 2 Theilen Kalilauge geschüttelt, so schied sich nach 24 Stunden eine ölartige Flüssigkeit $a b$, Cinnamein, welches auf einer braunen, kalihaltigen Flüssigkeit schwamm. Ilas Cinuanein, mit pulverisirten Kali und Schwefelkohlenstoff geschültelt, gals ein Salz, welches 
xanthogensaures Kali enthielt. Durch Destillation der von dem Cinnamein getrennten kalihaltigen Flüssigkeit wurde nur eine Spur von einem Oele erhalten; das übrige Destillat verhielt sich wie Wasser. Hieraus ergiebt sich also, dass schon bei gewöhnlicher Temperatur durch die Einwirkung von Kalilauge eine $\mathrm{Ab}$ sonderung der Verbindung vor sich geht, die sich bei einer passenden Wärme in Aetherarten umwandelt.

Wenn man den Perubalsam mit Wasser destillirt, so erhält man bekanntlich weder Oel noch Aether; da diess darin begründet sein konnte, daśs der Siedepunkt des Zimmtāthers zu hoch liegt, so destillirte ich den Balsam theils mit einer Losung von Kochsalz, theils mit einer von Zinkchlorid. Diese letzte Destillation wurde so ausgeführt, dass ich den Balsam in die kochende Lösung des Chlorzinks allmählig eintropfen liess. Hierbei destillirte ausser Wasser elwas Zinmtsāure über und eine äusserst geringe Menge von zwei braun gefärbten óligen Flüssigkeiten, von denen die eine schwerer, die andere leichter war als Wasser. Beide rochen brenzlich, und gaben beim Kochen mit Wasser nach dem Erkalten eine bedeutende Menge Zimmtsãurekrystalle. In dem Destillat konnte kein Zimmtäther entdeckt werden.

Unterwarf man den Perubalsam der trocknen Destillation, so wurden verschiedene ölige Flüssigkeiten erhalten, welche eine grosse Mlenge Zimmtsäure enthielten; ausserdem Wasser. Die beim Abkühlen auskrystallisirende Zimmtsäure wurde auf einem Filtrum gesammelt, und die Javon abgelaufene Flüssigkeit über Chlorcalcium getrocknet, überdestillirt, und sodann mit gepulvertem Kalihydrat und Schwefelkohlenstolf behandelt. In dem Gemenge ward keine Spur von Xanthogensäure entdeckt. Darauf wurde ein Theil Perubalsam mit einer Auflosung von kohlensaurem Natron behandelt; die Masse wurde zur Trockne eingedampft, unter Zusatz von trocknem kohlensaurem Natron und das Gemisch der trocknen Destillation unterworfen. Die hierbei gewonnenen Produkte waren im Aeussern denen der trocknen Destillation des Balsams für sich, ähnlich; aber es fanden sich, durch geeignete Behandlung, Spuren von Xanthogensăure.

Nach diesen Versuchen kann man nicht annehmen, dass 
der Perubalsam fertiggebildeten Zimmtälher enthalte, dass sich dieser jedoch durch Einwirkung starker Alkalien bilde. Wie bekannt hat $\mathrm{E}$. Simon bei seinen Untersuchungen über den flüssigen Storax einen Stoff angegeben, den er Styracon nannte; ich fand nun, dass, wenn man den flüssigen Storax auf dieselbe Weise behandelt wie den peruvianischen Balsam, man sogleich ein Destillat erhält, welches beim Stehenlassen einen Körper abscheidet, der die von Simon für das Styracon angegebenen Eigenscluaften besitzt. Ausserdem löst er sich in Weingeist von $84^{\circ} \mathrm{Tr}$. aul.

Bei der Behandlung des Styracons mit pulverisirtem Kali und Schwefelkohlenstoff, bildete sich unter andern xanthogensaures Kali. Ich vermuthete darnach, dass das Styracon einen zusammengesetzten Aether enthält, mit dessen genauerer Untersuchung ich beschältigt bin.

Aus Mangel an Styrol untersuchte ich nur das Verhalten des Benzols gegen Kali und Schwefelkohlenstoft, wobei sich jedoch kein xanthogensaures Kali bildete.

Darauf wurde Capaivabalsam mit kaustischem Kali behandelt, und sodann der Destillation unterworfen. Ich erlielt sogleich eine grosse Menge eines 0els, welches auf dem überdestillirten Wasser schwamm. Nachdem das Wasser zurückgegeben und von Neuem überdestillirt war, betrug die gesammelte Menge Oel etwa $10 \mathrm{Loth}$; das erhaltene Wasser ungefähr viermal soviel. Es war $1 \mathscr{t}$ Balsam und $1 \frac{1}{2} \mathbb{E}$ Kalilauge von 1,27 spec. Gew. angewendet worden. Das Oel war mit dem im Handel vorkommenden Capaivaōl identisch; bei Behandlung derselben mit Kali und Schwefelkohlenstoff zeigte sich nicht die Bildung von Xanthogensäure.

Da übrigens die Capaivasäure nicbt flüchtig ist, so habe ich die Hoffnung nicht aufgegeben, durch ein abgeändertes Verfahren aus diesem Balsam mittelst Kali einen Aether zu erhalten.

Als venetianischer Terpenthin auf ähnliche Weise behandelt wurde, erhielt man eine ölige Flüssigkeit, welche mit Chlorcalcium oder Kalk ausgetrocknet, von gepulvertem Kali nicht verändert zu werden schien. Die Farbe der Flüssigkeit blieb mehrere Stunden nach dem Zusatz des Kalis unverändert, und man hemerkte während der Mischung keine Temperatur-Erhöhung. Durch Zusatz von Schwefelkohlenstoff und Schütteln bildete sich 
nach einiger Zeit eine weisse Salzmasse, so dass nach und nach die ganze Mischung zu einer Gallerte erstarte, welche nach 24 Stunden auf das Filter gebracht wurde. Als der grösste Theil der flüssigen Masse alggelaufen war, wurde der olige Theil zwischen Papier abgepresst. Ein Theil der so erhaltenen Salzmasse wurde getrocknet und in Wasser aufgelöst; ein anderer Theil wurde mit Aether geschïttelt, um alles Harz und Oel daraus zu entfernen. Der Aether wurde abfiltrirt, und die auf dem Filtrum gesammelte Salzmasse getrocknet, und gleichfalls in reinem Wasser gelöst.

Beide Auflösungen brachten mit Kupferchlorid sogleich einen braunen Niederschlag hervor, der jedoch nach einiger Zeit gell, wurde. Mit salpetersaurem Bleioxyde wurde ein hellgelber Niederschlag erbalten, und mit verschiedenen anderen Salzen ähnliche Reaktion wie mit Xanthogensäure, indessen hatten alle diese Niederschläge eine etwas dunklere Farbe.

Diess Verhalten liess vermuthen, dass durch die Einwirknng des Kalis auf den venetianischen Terpenthin ein Aether erzeugt worden sei. Der Siedepunkt des Destillats war ungefähr $153^{\circ} \mathrm{C}$.; die Dichtigkeit 0,87. Die Löslichkeit in Weingeist von $93^{\circ} \mathrm{Tr}$. war gleich der des gewöhnlichen Terpenthinöls.

Dieses Verhalten stimmt so nahe mit dem des gewöhnlichen Terpenthinöls überein, dass die Vermuthung, die Flüssigkeit sei ein Aether, fort fällt; dagegen war es wahrscheinlich, dass das Oel, durch Destillation des venetianischen Terpenthins mit Wassers erhalten, gleiche Reaktionen gegen Kali und Schwefelwasserstoff zeigen würde, wie das oben angefïhrte durch Kali erhaltene. Der hierüber angestellte Versuch bestătigte diess; nun wurde gewöhnliches Terpenthinöl geprüf, welches frisch rectificirt und von Wasser befreit war, das Resultat war im Ganzen dasselbe. Aus reinem Terpenthinöl kann also mit Kalihydrat und Schwefelkohlenstoff eine Verbindung erzeugt werden, welche grosse Aehnlichkeit mit der Xanthogensäure hat. Damit jedoch dieser Körper entstehen kann, dazu ist durchaus nothwendig, dass das Kalihydrat gehörig geschmolzen sei; so wie es im Handel vorkommt, ist es noch zu wasserbaltig. Nicht die ganze Menge des Terpenthinöls kann in die Verbindung mit Sehwefelkohlenstoff und Kalihydrat übergeführt werden. Mit der Erzengung derseiben scheint es sich ähnlich zu verhalten 
wie mit der des Terpins, indem auch nur ein Theil des Oels in diese neue Verhindung übergeht.

Uebrigens bemerke ich hierbei, dass ungeachtet man gefunden haben will, dass jedes reine Terpenthinöl aus $\mathrm{C}_{10} \mathrm{II}_{3}$ bestehe, und der Siedepunkt und das spec. Gervicht davon gleich sei, doch das Oel des venetianischen Terpenthins von dem gewöhnlichem in mehrerer Ilinsicht verschieden ist; so wird das letzlere bei der Einwirknng des lialis schnell gefärbt, was bei jenem nicht der FiH ist. Das Oel des venetianischen Terpenthins polarisirt das Licht nach links; jedoch ist um die violelte Farbe zu erbalten, nur eine Umdrelung ron $2 \check{5}^{n}$ erforderlich. Eljenso verhielt sich eine gessätigte Auflösung von salzsaurem Dadyl in Weingeist, welches aus diesem 0el bereitet worden war.

Gewöhnliches Terpenthinöl polarisirt gleichfalls das Licht nach links; erfordert jedoch um den violetten Strahl zu erhalten, eine Drehung von 67 bis $70^{\circ}$. Ebenso verhält sich sowohl eine gesälligte alkoholische Lösung des chlorwasserstoffsauren Dadyls aus demselben, als auch der Rest des Oels, aus dem Schwefelkohlenstoff und Kalihydrat die der Xanthogensäure äınliche Verbindung ausgeschieden hatte.

Wenn man das Terpenthinöl länger und schleclit aufbewalıt hat, so wirkt das Kalibydrat um so stārker darauf ein. Es tritt dann zuweilen eine Wärmeentwickelung von mehr als $60^{\circ}$ ein, wobei sich cine braune gallertarlige Masse bildet, welche in hohem Grade, der Erzeugung der der Xanthorensäure äbnlichen Verbindung widersteht. Selbst wenn man kürzlich rectificirtes und durch Kalk entwässertes Terpenthinül anwendet, so erhält man doch meist ein viel dunkleres Präparat, als wenn man das Oel des venetianischen Terpenthins benutzt. Lässt man die entstandene Salzmasse, welche in der Regel theils freies Kali theils Schwefelkalium enthült, an der Luft liegen, so wird sie heller und liefert mit Kupferoxydsalzen einen Niederschlag, der schneller gelb wird. Aus Terpin, Schwefelkohlenstoff und Kalihydrat habe ich niclst die xanthogenartige Verbindung erhalten können, ob es mit Terpinol glücken wird, habe ich noch nicht untersuchen können. Ebenso werde ich künftig noch das Verhalten anderer atherischen 0ele prüfen. -

Mittlerweile geht aus dem Angeführten hervor, dass meh- 
rere frühere Erfahrungen über einige dieser Gegenstände, welche vereinzelt dastanden, nun in einem allgemeinen Zusammenhang sich zeigen, und dass die Untersuchungen von $Z$ eis e äber die Alkohol-Xanthogensăure eine grössere Wichtigkeit erhalten, indem die Wechselwirkung zwischen Kali, Schwefelkohlenstoff und kohlenwasserstofthaltigen Körpern sich nicht auf wenige Verbindungen, sondern auf ganze Klassen erstreckt.

Dass die angeführten Untersuchungen zur Entwickelung umfassenderer Aetherbildungstheorien dienen werden, kann wohl nicht bezweifelt werden.

\section{Literatur.}

Lehrbuch der Chemie von 0 tto Linné Erdmann. Vierte vollig umgearbeitete und vermehrte Auflage. Erste Lief. Bogen 1-24. (Enthält die unorganische Chemie. Die zweite und letzte Lief. Corganische Chemie) erscheint vor Schluss des Jahres.) Leipzig 1850. Verlag von J. A. Barth.

Die unorganische Chemie, ein Grundriss für seine Vorlesungen an der Artillerie- und Ingenieur-Schule zu Berlin. Von Dr. G. Werther. Erste Abtheilung: Die unorganische Chemie mit Ausnahme der zur Artillerie - und Ingenieur-Technik gehörigen Gegenstände. Berlin. Druck und Verlag von G. Reimer. 1850.

Commentar zur Preussischen Pharmacopoe, nebst Uebersetzung des Textes. Von Dr. F. Mohr pp. Sechste Lief. Braunschweig bei F. Viewe $\mathrm{g}$ and Sohn. 1850.

Ueber die Maassanalysen, besonders in ihrer Anwendnng auf die Bestimmung des technischen Werthes der chemischen Handelsprodukte, wie Pottasche, Soda, Chlorkalk, Braunstein, Săuren, Arsen, Chrom, Eisen, Kupfer, Zinn, Blei, Silber u. s. w. Von Er. H. Schwarz, Privatdocenten der Ghemie an der Universitït zu Breslau. Mit Holzschnitten. Braunschweig Druck und Verlag von F. Vieweg und Sohn. 1850.

Jahresbericht uber die Fortsohritte der reinen pharmaceutischen und technischen Chemie, Physik, Mineralogie und Geologie. Onter Mitwirkung von Buff, Dieffenbach, Ettling, Knapp, Will, Zamminer, herausgegeben von Justus Liebig und Hermann Ko p p. Für 1849. Erstes Heft. (Ausgegeben am 29. Juni 1850.) Zweites Heft. (Ausgegeben am 31. Aug. 1850.) Giessen. J. Ricker'sche Bachhandlung. 1850. 